\title{
In Vivo Imaging of Human Cholinergic Nerve Terminals with (-)-5-18 F-Fluoroethoxybenzovesamicol: Biodistribution, Dosimetry, and Tracer Kinetic Analyses
}

\author{
Myria Petrou ${ }^{1}$, Kirk A. Frey ${ }^{1,2}$, Michael R. Kilbourn ${ }^{1}$, Peter J.H. Scott ${ }^{1}$, David M. Raffel ${ }^{1}$, Nicolaas I. Bohnen ${ }^{1-3}$, \\ Martijn L.T.M. Müller ${ }^{1}$, Roger L. Albin ${ }^{2,3}$, and Robert A. Koeppe ${ }^{1}$ \\ ${ }^{1}$ Department of Radiology, Division of Nuclear Medicine, University of Michigan Medical School, Ann Arbor, Michigan; ${ }^{2}$ Department \\ of Neurology, University of Michigan Medical School, Ann Arbor, Michigan; and ${ }^{3}$ Neurology Service and GRECC, VAAAHS, \\ Ann Arbor, Michigan
}

$(-)-5-{ }^{18} \mathrm{~F}$-fluoroethoxybenzovesamicol $\left({ }^{18} \mathrm{~F}-\mathrm{FEOBV}\right)$ is a vesamicol derivative that binds selectively to the vesicular acetylcholine transporter (VAChT) and has been used in preclinical studies to quantify presynaptic cholinergic nerve terminals. This study presents, to our knowledge, the first-in-human experience with ${ }^{18} \mathrm{~F}-\mathrm{FEOBV}$, including radiation dosimetry, biodistribution, tolerability and safety in human subjects, and brain kinetics and methods for quantitative analysis of ${ }^{18} \mathrm{~F}-\mathrm{FEOBV}$. Methods: Whole-body ${ }^{18} \mathrm{~F}-\mathrm{FEOBV}$ scans were obtained in 3 healthy human volunteers. Seven additional subjects underwent dynamic brain imaging $0-120,150-180$, and 210$240 \mathrm{~min}$ after bolus injection of ${ }^{18} \mathrm{~F}-\mathrm{FEOBV}$. Arterial blood sampling was performed with chromatographic identification of authentic ${ }^{18} \mathrm{~F}$ FEOBV to determine the arterial plasma input function. Analysis methods included nonlinear least-squares fitting of a 2-tissuecompartmental model, reference tissue modeling, and late singlescan imaging. Results: No pharmacologic or physiologic changes were observed after intravenous administration of up to $1.3 \mu \mathrm{g}$ of ${ }^{18} \mathrm{~F}-\mathrm{FEOBV}$. Radiation dosimetry estimates indicate that more than $400 \mathrm{MBq}$ may be administered without exceeding regulatory radiation dose limits. Kinetic analysis showed brain uptake to be relatively high with single-pass extraction of $25 \%-35 \%$. VAChT binding estimates varied by a factor of greater than 30 between the striatum and cortex. Coefficients of variation in $k_{3}$ estimates varied from $15 \%$ to $30 \%$. Volume of distribution measures yielded a dynamic range of approximately 15 but with little reduction in variability. Reference tissue approaches yielded more stable estimates of the distribution volume ratio $\left(1+B P_{\mathrm{ND}}\right)$, with coefficients of variation ranging from $20 \%$ in the striatum to $6 \%-12 \%$ in cortical regions. The late static distribution of ${ }^{18} \mathrm{~F}-\mathrm{FEOBV}$ correlated highly with the distribution volume ratio estimates from reference tissue models $(r=0.993)$. Conclusion: ${ }^{18} \mathrm{~F}-\mathrm{FEOBV}$ PET confirms that the tracer binds to VAChT with the expected in vivo human brain distribution. Both reference tissue modeling and late static scanning approaches provide a robust index of VAChT binding.

Key Words: ${ }^{18} \mathrm{~F}-\mathrm{FEOBV}$; PET; VAChT; dementia

J Nucl Med 2014; 55:396-404

DOI: 10.2967/jnumed.113.124792

Received Apr. 13, 2013; revision accepted Oct. 10, 2013.

For correspondence contact: Robert A. Koeppe, University of Michigan, 2276 Med Sci I, SPC 5610, 1301 Catherine, Ann Arbor, Ml 48109-5610.

E-mail: koeppe@umich.edu

Published online Jan. 30, 2014.

COPYRIGHT (c) 2014 by the Society of Nuclear Medicine and Molecular Imaging, Inc.
$\mathbf{T}$ he noninvasive measurement of regional brain cholinergic terminal integrity was made possible by the introduction of the SPECT radiotracer (-)-5- ${ }^{123}$ I-iodobenzovesamicol ( ${ }^{123}$ I-IBVM) (1). ${ }^{123}$ I-IBVM imaging showed declines in regional cholinergic terminal density in normal aging, Alzheimer disease (AD), Parkinson disease (PD), and dystonia (2,3). An analogous positron-emitting radiotracer would capitalize on the physical advantages of PET and potentially offer improved quantification of cholinergic projection system integrity.

Brain cholinergic systems include basal forebrain corticopetal projections, striatal interneurons, widespread projections from the brain stem pedunculo-pontine-laterodorsal tegmental complex (PPN), and the medial habenular-interpeduncular nucleus projection. Cholinergic systems are crucial in an array of important central nervous system (CNS) functions, including attentional and executive function, different forms of learning, regulation of sleep-wake cycles, and maintenance of posture and gait. The degeneration of cholinergic systems is implicated in clinically important features of several neurodegenerative disorders, including $\mathrm{AD}, \mathrm{PD}$, and progressive supranuclear palsy. Improved in vivo characterization of human brain cholinergic systems is important for improving the understanding and treatment of clinical deficits in these neurologic disorders.

Targets for in vivo imaging of brain cholinergic terminals include the acetylcholine (ACh)-degrading enzyme acetylcholinesterase (AChE) and the vesicular ACh transporter (VAChT). Prior in vivo PET imaging methods for quantifying cholinergic terminals rely on tracers that are $\mathrm{AChE}$ substrates, such as

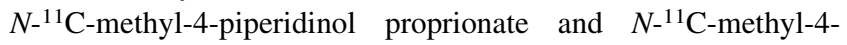
piperidinol acetate. Although AChE activity generally is correlated with cholinergic terminal distribution, some noncholinergic neurons express $\mathrm{AChE}$, and there are regions, such as the cerebellar hemispheres, in which high AChE expression is apparently decoupled from cholinergic innervation $(4,5)$. Vesamicol and its derivatives bind to VAChT at a vesamicol receptor site, proposed to be distinct from the ACh binding site. To extend human VAChT imaging studies to a higher level of precision with PET imaging, we developed a novel ${ }^{18} \mathrm{~F}$-labeled ligand, $(-)-5-{ }^{18} \mathrm{~F}$-fluoroethoxybenzovesamicol ( ${ }^{18} \mathrm{~F}$-FEOBV; Fig. 1) (6).

Our prior studies in rodents and nonhuman primates demonstrated that ${ }^{18} \mathrm{~F}$-FEOBV is a reliable imaging tool for VAChT in mammalian brain. Ex vivo dissection and small-animal PET 


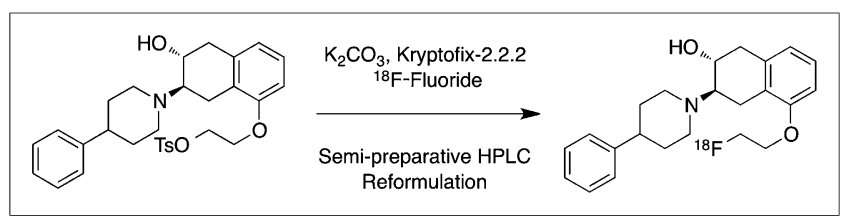

FIGURE 1. FEOBV structure and synthesis path.

imaging studies of rodent brain revealed distribution and retention of ${ }^{18} \mathrm{~F}$-FEOBV consistent with the expected distribution of cholinergic terminals, showing differential binding in the cortex, cerebellum, and striatum (7). PET imaging studies in nonhuman primate brain also demonstrated heterogeneous binding and retention in a pattern expected from rodent data (7).

Preliminary human dosimetry estimates based on extrapolation from rodent data suggest that at an administered dose of $240 \mathrm{MBq}$ $(6.5 \mathrm{mCi})$, the effective radiation dose will be $12.9 \mathrm{mSv}$, and exposure of individual organs would fall under acceptable limits for clinical research in the United States. In preclinical toxicity studies, rats were observed after injection of FEOBV at 80 and 1,000 times the proposed maximum human mass dose $(0.0175 \mu \mathrm{g} / \mathrm{kg})$. No adverse effects were observed during a 15-d follow-up period after FEOBV injection of $1.40 \mu \mathrm{g} / \mathrm{kg}, 80 \times$ the proposed human dose. Only transient effects not considered to be dose-limiting toxic effects were observed in the first $24 \mathrm{~h}$ after dose administration at the $1,000 \times$ dose $(17.6 \mu \mathrm{g} / \mathrm{kg})$. These were slight hypoactivity in 5 of 10 animals and slight to moderate tremors in the head and thoracic region in 6 of 10 animals. Cumulative animal studies data indicate that ${ }^{18} \mathrm{~F}$-FEOBV binds VAChT with adequate sensitivity and specificity and suggests appropriate dosimetry and toxicology profiles for human applications.

The primary objectives of the current study were to estimate radiation dosimetry and biodistribution, to assess tolerability and safety of ${ }^{18}$ F-FEOBV in human subjects, and to measure the brain kinetics and assess methods for quantitative analysis of ${ }^{18}$ F-FEOBV.

\section{MATERIALS AND METHODS}

\section{Study Population}

All volunteers participating in the study were over the age of $18 \mathrm{y}$. Three subjects (mean age, $36 \mathrm{y}$; range, 18-56 y; 2 women, 1 man) underwent total-body imaging for determination of radiotracer dosimetry. Seven subjects (mean age, 45 y; range, 20-66 y; 2 women, 5 men) underwent dynamic brain imaging. Subjects had no prior history of neurologic or psychiatric illness and had normal general physical and neurologic examinations. Women with childbearing potential were not pregnant (negative urine $\beta$-human chorionic gonadotropin on day of tracer administration). Additional exclusion criteria included breastfeeding, anticoagulant medication, antiplatelet agents used in the $5 \mathrm{~d}$ before the imaging visit, contraindications to MR imaging, or any serious medical condition that might increase risk to the subject if they participated in the study.

\section{Study Design}

The experimental procedures were approved by the University Institutional Review Board and the subcommittee overseeing the use of radionuclides in humans. All subjects gave written informed consent before study participation. Subjects were screened within $30 \mathrm{~d}$ before PET, including recording of demographic information, clinical history, concomitant medications, physical and neurologic examination, and neuropsychologic testing. Structural brain MR imaging was performed before PET.
${ }^{18} \mathrm{~F}-\mathrm{FEOBV}$ was prepared as described previously (8). The average yield of ${ }^{18} \mathrm{~F}-\mathrm{FEOBV}$ was $5,400 \mathrm{MBq}(146 \mathrm{mCi})$ ( $~ 9 \%$ non-decaycorrected yield), and average specific activity was $166 \mathrm{TBq} / \mathrm{mmol}$ (4,500 Ci/mmol). ${ }^{18} \mathrm{~F}-\mathrm{FEOBV}(238-260 \mathrm{MBq})$ was administered by intravenous bolus. Subjects were scanned with eyes and ears unoccluded in a dimly lit scanning environment using an ECAT Exact HR + PET tomograph (Siemens Molecular Imaging, Inc.).

\section{Whole-Body PET Imaging}

${ }^{18} \mathrm{~F}$-FEOBV imaging in 3-dimensional mode spanned the top of head to mid thigh. Four sequential whole-body images were acquired: approximately 4-56, approximately 58-110, approximately 160-212, and approximately 360-412 min after injection. Each scan consisted of 7 bed positions: 7-min acquisition per bed position (4-min 3-dimensional emission and 3-min 2-dimensional transmission scans per bed position) requiring additional time for insertion and retraction of the interplane septa.

Regions of interest were defined for the following structures: urinary bladder, kidneys, gastrointestinal tract, spleen, gallbladder, liver, bone (vertebral spine, sternum, clavicle, scapula), left ventricle, lower lung, upper lung, thyroid, parotid, brain, and whole body.

Dosimetry estimates were generated from the organ time-activity data using the OLINDA/EXM software package (9). For most organs, decay-corrected time-activity data were entered into the Disintegrations Calculator feature of OLINDA/EXM to calculate the cumulated activity (MBq-hr/MBq) occurring in each source organ. Because of their more complex kinetics, the cumulated activity values for the liver and gallbladder were calculated using the software package Prism 3 (GraphPad, Inc.). The uptake and clearance kinetics for the organs were fitted to an appropriate model, had ${ }^{18} \mathrm{~F}$ decay factors applied, and were numerically integrated to provide an estimate of the cumulated activity. Values were entered into the Kinetics Input Form of OLINDA/EXM. Data for the increasing activity in the bladder contents were fitted to a model containing exponential terms to determine the appropriate fractions and half-lives for the Voiding Bladder Model

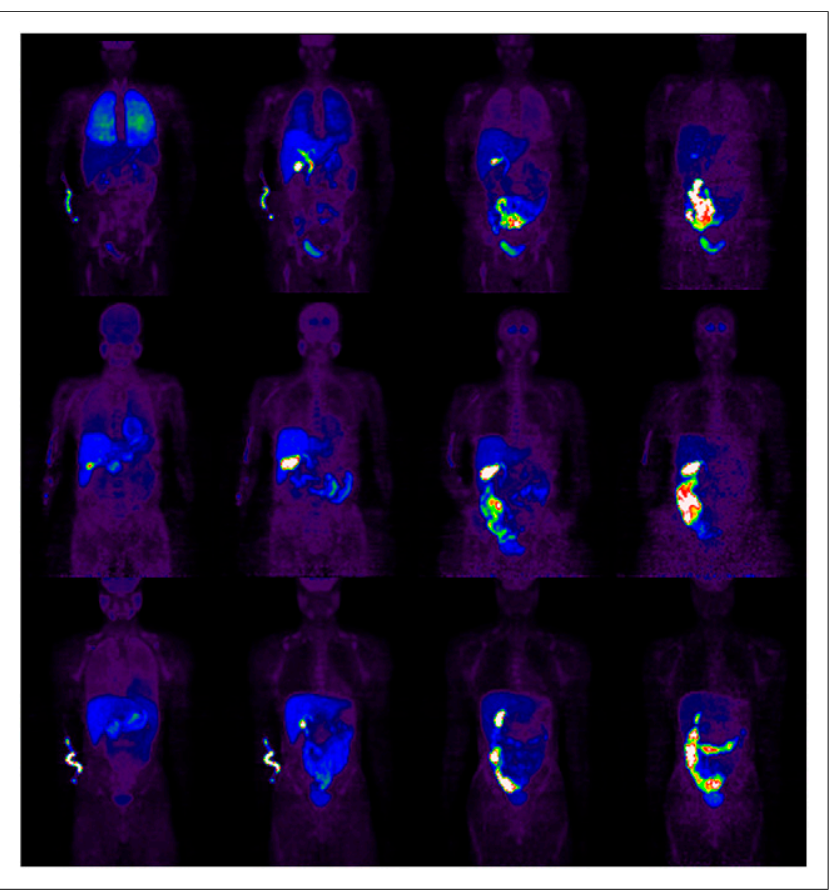

FIGURE 2. Whole-body PET images of ${ }^{18} \mathrm{~F}-\mathrm{FEOBV}$ in 3 healthy controls. Start times for each of four 50-min acquisitions are (from left to right) $5,60,160$, and $360 \mathrm{~min}$. Each scan is decay-corrected to time of injection. 
TABLE 1

${ }^{18}$ F-FEOBV Human Dosimetry Estimates

\begin{tabular}{lcrr}
\hline \multicolumn{1}{c}{ Organ } & Mean mGy/MBq & SD & $\mathrm{mGy} / 450 \mathrm{MBq}$ \\
\hline Lens of eye & $1.36 \times 10^{-2}$ & $1.34 \times 10^{-3}$ & 6.1 \\
Red marrow & $1.31 \times 10^{-2}$ & $7.38 \times 10^{-4}$ & 5.9 \\
Ovary & $2.14 \times 10^{-2}$ & $1.05 \times 10^{-3}$ & 9.6 \\
Testis & $7.05 \times 10^{-3}$ & $7.20 \times 10^{-4}$ & 3.2 \\
Lower large intestine & $3.61 \times 10^{-2}$ & $1.39 \times 10^{-3}$ & 16.3 \\
Upper large intestine & $1.01 \times 10^{-1}$ & $3.12 \times 10^{-3}$ & 45.4 \\
Small intestine & $8.90 \times 10^{-2}$ & $2.67 \times 10^{-3}$ & 40.1 \\
Liver & $4.60 \times 10^{-2}$ & $1.84 \times 10^{-3}$ & 20.7 \\
Gallbladder & $9.91 \times 10^{-2}$ & $5.13 \times 10^{-3}$ & 4.6 \\
Lung & $3.05 \times 10^{-2}$ & $2.26 \times 10^{-2}$ & 13.7 \\
Effective dose & $2.26 \times 10^{-2}, \mathrm{mean} \mathrm{mSv} / \mathrm{MBq}$ & $2.16 \times 10^{-3}, \mathrm{SD}$ & $10.2, \mathrm{mSv} / 450 \mathrm{MBq}$ \\
\hline
\end{tabular}

of OLINDA/EXM. A 4-h bladder-voiding interval was assumed. The kinetics of ${ }^{18} \mathrm{~F}-\mathrm{FEOBV}$ in the gastrointestinal tract were also modeled using exponential terms, and the results used to estimate the percentage of the injected dose excreted through this pathway. Dosimetry estimates were generated for the Reference Adult Male model of OLINDA/EXM.

\section{Safety and Tolerability Evaluation}

Physical and neurologic examinations were performed at the imaging visit immediately before ${ }^{18} \mathrm{~F}-\mathrm{FEOBV}$ administration. Vital signs were recorded, electrocardiograms performed, and blood and urine samples collected. Measures included a comprehensive metabolic panel, lipid profile, a complete blood count, and a urinalysis. Vital signs were recorded at approximately 90 and $180 \mathrm{~min}$ after ${ }^{18} \mathrm{~F}-\mathrm{FEOBV}$ administration. Postadministration physical and neurologic examinations, vital signs, blood and urine analyses, and repeated electrocardiograms were performed approximately $390 \mathrm{~min}$ after ${ }^{18} \mathrm{~F}-\mathrm{FEOBV}$ administration.

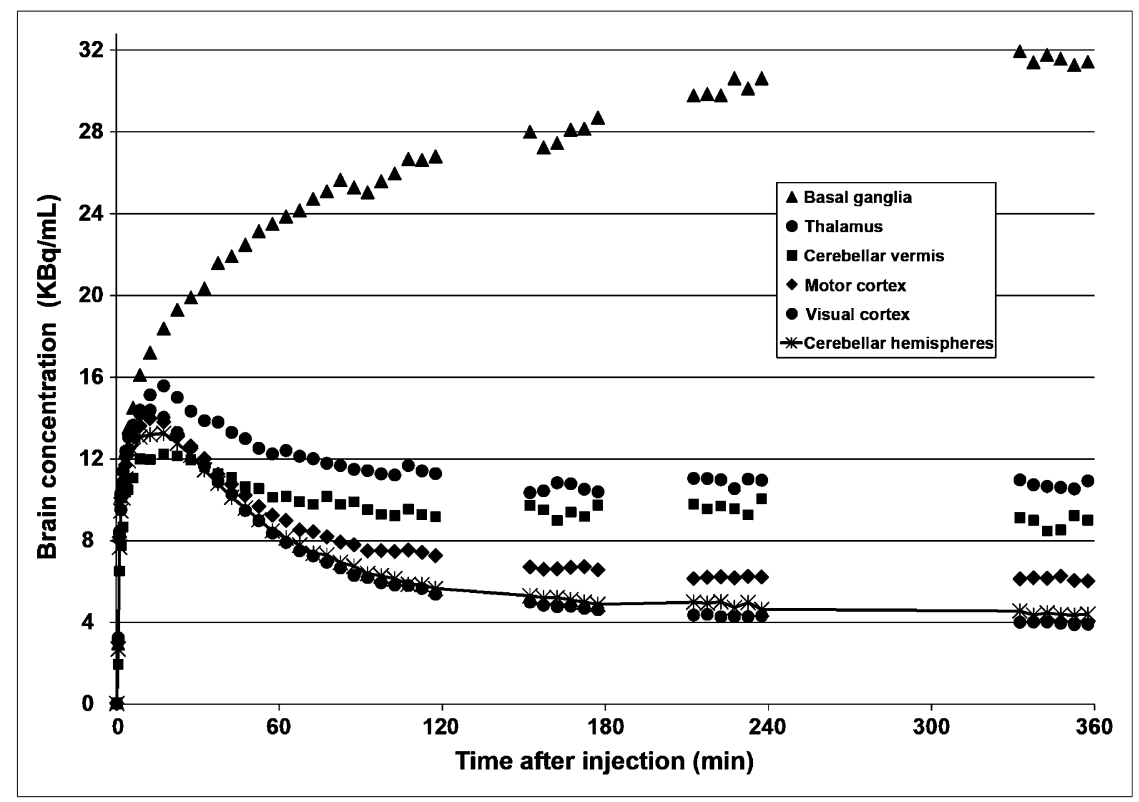

FIGURE 3. Tissue time-activity curves. ${ }^{18} \mathrm{~F}-\mathrm{FEOBV}$ concentrations are shown for several brain regions from scan in 1 healthy control subject. Although all regions have some VAChT, cerebellar hemispheres (shown with connecting line), a region likely to be largely unaffected in dementia, was used as reference region in all reference tissue approaches and as normalizing region for static late-frame analyses. For this subject, standardized uptake value (SUV) of 1.0 is approximately $3.30 \mathrm{kBq} / \mathrm{mL}$, and hence SUV in striatum has SUV of between 9 and 10 .

\section{Dynamic Brain Imaging}

Brain imaging was conducted in 3-4 imaging periods. The first period began at injection and continued for $120 \mathrm{~min}$. The subjects were then given a 30-min break, followed by a second imaging period from 150 to $180 \mathrm{~min}$, another break, and a third imaging period from 210 to $240 \mathrm{~min}$. In 2 subjects, an additional scan session was acquired from 5.5 to $6.0 \mathrm{~h}$. Arterial blood samples were obtained at multiple time points from a radial arterial cannula contralateral to tracer injection sites. Samples were taken every $10 \mathrm{~s}$ after ${ }^{18} \mathrm{~F}-\mathrm{FEOBV}$ administration during the first $2 \mathrm{~min}$ and subsequently at $3,4,5,7.5,10,15$, 20, 30, 40, 60, 90, 120, 180, 240, and $360 \mathrm{~min}$. Metabolite analysis was applied to arterial samples collected at the 2, 5, 10, 30, 60, 120, and 240 min time points. After centrifugation of the arterial samples, authentic ${ }^{18} \mathrm{~F}$-FEOBV was extracted from plasma into ethyl acetate using 3 extraction steps, whereas the polar metabolites remained in plasma (6). The combined organic phases and the residual aqueous phase were assayed in a NaI well counter. Dynamic PET scans were reconstructed using Fourier rebinning and iterative reconstruction (2-dimensional ordered-subset expectation maximization), 4 iterations, 16 subsets. No smoothing was applied.

\section{Kinetic Analyses}

Three general analytic approaches were evaluated. First, arterial input-based kinetic modeling was performed using a 2-tissuecompartment configuration, determining the standard rate parameters, $K_{1}-k_{4}$. Several variations in the general 2-tissue-compartment configurations were examined: estimation of all 4 rate constants; estimation of 3 rate constants with a fixed value for the dissociation rate constant, $k_{4}$; estimation of 3 rate constants with a fixed value for the nondisplaceable volume of distribution, given by the ratio, $K_{1} / k_{2}$; and simultaneous fitting of multiple time-activity curves for a given subject, with the constraint that $K_{1} / k_{2}$ or $k_{4}$ has the same value for all regions for that subject. The VAChT binding indices examined included $k_{3}$; the total volume of distribution, $V_{\mathrm{T}}$, given kinetically by $\left(K_{1} / k_{2}\right)\left[1+k_{3} / k_{4}\right]$; and the binding potential (relative to nondisplaceable uptake), $B P_{\mathrm{ND}}$ (either directly by the ratio $k_{3} / k_{4}$ or indirectly using a reference tissue by the ratio of $V_{\mathrm{T}}$ estimates in the target and reference regions - 1). Estimates were obtained using 


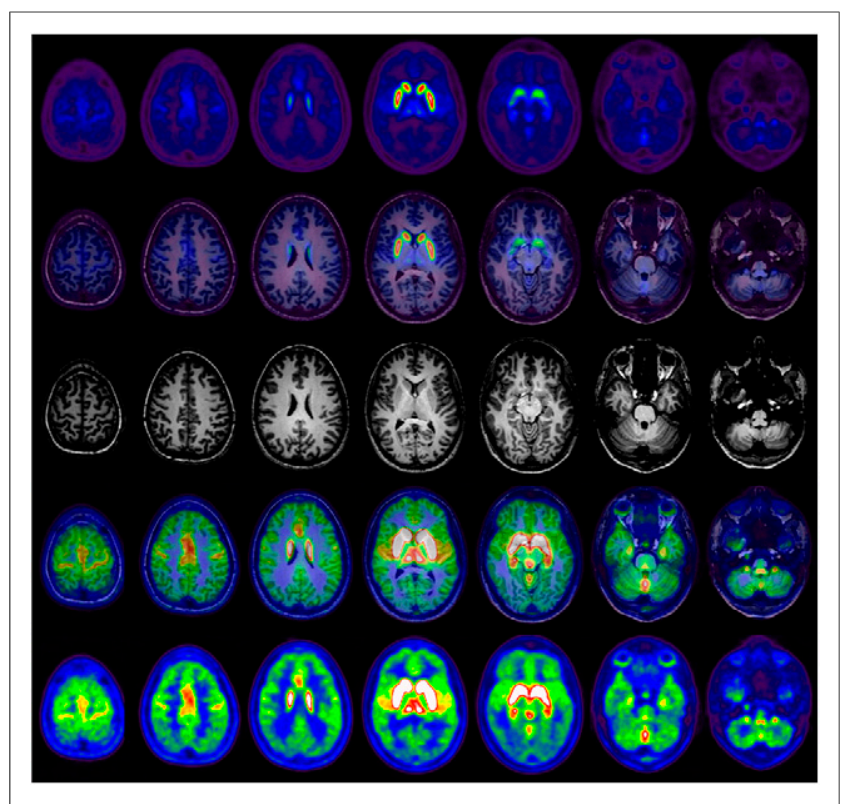

FIGURE 4. PET, MR, and overlay images from healthy control subject. Top and bottom rows are late summed ${ }^{18}$ F-FEOBV images $(3.5-4.0+$ 5.5-6.0 h). Bottom row shows same images as in top row, except with upper window scaled to $30 \%$ of maximum. Second and fourth rows are overlays of MR image.

the full image sets and also using shorter image durations of 120 and $90 \mathrm{~min}$.

Second, reference tissue methods, including Logan (10), simplified reference tissue model (sRTM) (11), and multilinear reference tissue model (mRTM) (12), were explored using cerebellar gray matter as the reference tissue, giving estimates of the $B P_{\mathrm{ND}}$. Estimates were generated using the full dynamic sequence (extrapolating values for the miss-

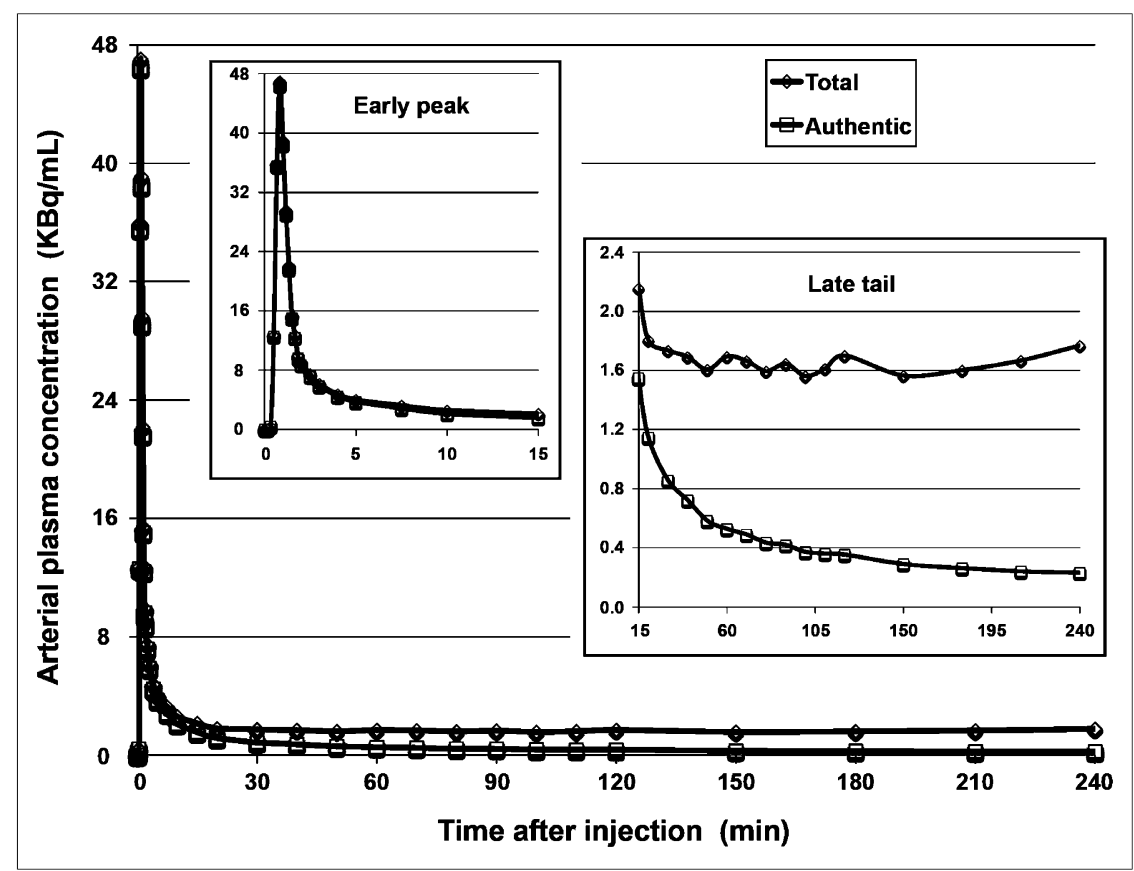

FIGURE 5. Arterial plasma input function. Concentration of both total radioactivity and authentic ${ }^{18} \mathrm{~F}-\mathrm{FEOBV}$ is shown for 1 control subject. Inlays show early and late sections of arterial plasma curve. ing time periods) and 3 shorter periods, 120, 90, and $60 \mathrm{~min}$. Both volume of interest and voxel-based analyses were conducted. For voxelwise analyses, a fixed value for the reference tissue $k_{2}$ was used (sRTM2 and mRTM2). Values for $k_{2}$ were obtained both from the kinetic estimates and from the estimates obtained from SRTM and $\mathrm{mRTM}$.

Third, simple late static summed images were created from the 2.5to 3.0 -h plus 3.5 - to 4.0 -h scans. The single summed image was normalized to cerebellar gray matter as in the reference tissue method calculations.

\section{RESULTS}

\section{Safety}

${ }^{18} \mathrm{~F}-\mathrm{FEOBV}$ administration was well tolerated by all 10 subjects. Administered mass dosages of FEOBV were $0.7 \pm 0.3 \mu \mathrm{g}$ (range, 0.3-1.3). No alterations were noted in vital signs or in physical or neurologic examinations, nor were significant changes noted in either electrocardiograms or any of the laboratory values.

\section{Biodistribution and Radiation Dosimetry}

${ }^{18} \mathrm{~F}-\mathrm{FEOBV}$ distributed rapidly throughout the body, including the CNS. ${ }^{18}$ F-FEOBV was excreted primarily via the hepatobiliary system, with tracer accumulation evident in the gallbladder, small intestine, and large intestine in whole-body images (Fig. 2). Radiation doses to organs (Table 1) fell within the range predicted by preclinical rodent studies. On the basis of preliminary human dosimetry from 3 subjects' PET scans, injected doses of up to $450 \mathrm{MBq}(\sim 12 \mathrm{mCi})$ would not exceed U.S. Food and Drug Administration-permitted limits of $50 \mathrm{mSv}$ to the critical organs (gallbladder, small intestine, and large intestine) or $30 \mathrm{mSv}$ to the whole body or radiosensitive organs.

\section{Brain Kinetics, Compartmental Modeling, and VAChT \\ Binding Estimates}

The range of ${ }^{18} \mathrm{~F}$-FEOBV kinetics in the human brain is seen in Figure 3. Initial brain uptake demonstrated a gray matter pattern, consistent with cerebral blood flow-mediated tracer delivery and extraction at the blood-brain barrier. By $30 \mathrm{~min}$ after injection, tracer retention began to demonstrate the expected distribution of cholinergic nerve terminals, with progressively increasing contrast between structures with known high (striatum) and low (lateral cerebellar hemisphere cortex) cholinergic innervation. By 30-60 min after injection, tracer levels peaked and demonstrated clearance from brain regions with moderate to low cholinergic innervation (thalamus, cerebellar vermis, cerebral cortex). In the striatum, the region of highest cholinergic terminal density, tracer continued to accumulate throughout the entire study. In all regions, ${ }^{18}$ F-FEOBV concentrations were relatively constant after $3 \mathrm{~h}$, suggesting predominance of VAChTbound ligand (with slow dissociation kinetics), or alternatively, rapid rebinding of dissociating tracer. 


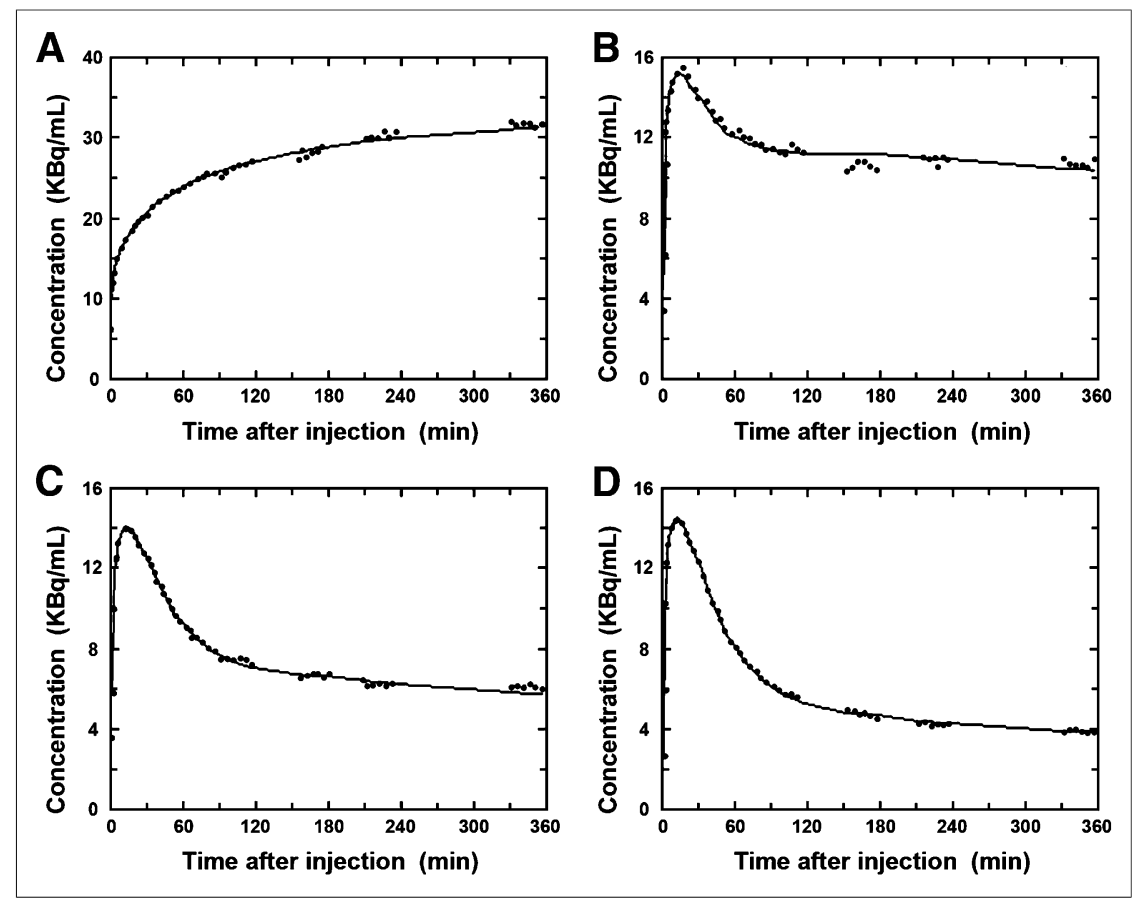

FIGURE 6. Nonlinear least-squares fits to ${ }^{18} \mathrm{~F}-\mathrm{FEOBV}$ time-activity curves. Simultaneous fitting of multiple regions was performed on time-activity curves. In single combined fit, region-specific $K_{1}$ and $k_{3}$ were estimated for each curve, whereas single values for all regions were estimated for $K_{1} / k_{2}\left(=V_{\text {ND }}\right.$ [volume of distribution of the nondisplaceable component of ${ }^{18} \mathrm{~F}-\mathrm{FEOBV}$ uptake]) and dissociation rate constant, $k_{4}$. (A) Putamen. (B) Thalamus. (C) Motor cortex. (D) Cerebellum.

with the location of the PPN complex. In the cerebellar cortex, binding was particularly marked in the vermis, flocculus, and nodulus.

All subjects showed rapid clearance of radioactivity from arterial plasma over the first 5 min of the study, followed by slower clearance over the next $4 \mathrm{~h}$. Final concentrations of authentic ${ }^{18} \mathrm{~F}-\mathrm{FEOBV}$ in arterial plasma were less than $1 \%$ of the early peak concentration. Analysis of arterial blood samples for radiolabeled metabolites showed that the fraction of parent ${ }^{18} \mathrm{~F}$ FEOBV in arterial plasma dropped to about $50 \%$ by $30 \mathrm{~min}$ after injection and then continued to decline slowly for the remainder of the study, ranging from $9 \%$ to $18 \%$ (mean $\pm \mathrm{SD}, 12.8 \% \pm 3.2 \%$ ) at $4 \mathrm{~h}$ after injection. Correction of the arterial plasma for radiolabeled metabolites yields the plasma concentration time course of ${ }^{18} \mathrm{~F}-\mathrm{FEOBV}$ used for compartmental modeling (Fig. 5).

Kinetic analyses were performed using a 2-tissue-compartmental model configuration. Nonlinear least-squares fits of volume-of-interest time-activity curves using the metabolite-corrected arterial input function were performed on multiple time-activity curves (Fig. 6). Compart-

The dynamic range of ${ }^{18} \mathrm{~F}-\mathrm{FEOBV}$ activity in the brain at $3.5 \mathrm{~h}$ after injection is large (Fig. 4), with striatal radioactivity concentrations 5-6 times higher than in cerebral and cerebellar cortices. Within some brain structures, there were interesting subregional variations in ${ }^{18} \mathrm{~F}-\mathrm{FEOBV}$ retention. In the neocortex, areas of higher tracer retention included the anterior cingulate, supplementary motor area, and primary sensorimotor cortex. Relatively high tracer retention was also seen in the medial-anterior temporal lobe, suggesting VChAT expression in the hippocampal formation or amygdala. In the brain stem, there was considerable tracer retention in the medulla and at the mesopontine junction, consistent mental modeling when fitting 4 rate parameters yielded VChAT estimates with poor precision $\left(k_{3}\right.$ coefficient of variation $>50 \%$; results not shown). Modest improvements were seen when constraining the dissociation rate constant, $k_{4}$, or the nondisplaceable volume of distribution, $K_{1} / k_{2}$, ( $k_{3}$ coefficient of variation, 35\%$40 \%)$. The most robust VChAT estimates were obtained by estimating region-specific values for the blood-brain barrier transport rate constant $\left(K_{1}\right)$ and the rate constant for transporter binding $\left(k_{3}\right)$ simultaneously across all regions, while using common values (though different for each subject) for the ratio of $K_{1} / k_{2}$; the dissociation rate constant, $k_{4}$; the cerebral blood volume; and the time

TABLE 2

Compartmental Modeling Parameter Estimates

\begin{tabular}{|c|c|c|c|c|c|c|c|c|c|c|}
\hline \multirow[b]{2}{*}{ Subject } & \multicolumn{4}{|c|}{$K_{1}$} & \multirow[b]{2}{*}{$\begin{array}{c}K_{1} / k_{2}, \\
\text { all }\end{array}$} & \multicolumn{4}{|c|}{$k_{3}$} & \multirow[b]{2}{*}{$k_{4}$, all } \\
\hline & Striatum & Thalamus & $\begin{array}{l}\text { Motor } \\
\text { cortex }\end{array}$ & $\begin{array}{l}\text { Cerebellar } \\
\text { gray matter }\end{array}$ & & Striatum & Thalamus & Motor cortex & $\begin{array}{l}\text { Cerebellar } \\
\text { gray matter }\end{array}$ & \\
\hline 1 & 0.343 & 0.388 & 0.249 & 0.287 & 9.45 & 0.117 & 0.0173 & 0.0076 & 0.0044 & 0.0045 \\
\hline 2 & 0.216 & 0.232 & 0.183 & 0.202 & 5.93 & 0.178 & 0.0167 & 0.0068 & 0.0047 & 0.0054 \\
\hline 3 & 0.215 & 0.200 & 0.144 & 0.197 & 4.24 & 0.073 & 0.0135 & 0.0102 & 0.0037 & 0.0048 \\
\hline 4 & 0.321 & 0.319 & 0.280 & 0.328 & 7.02 & 0.092 & 0.0162 & 0.0056 & 0.0029 & 0.0028 \\
\hline 5 & 0.227 & 0.201 & 0.156 & 0.199 & 6.47 & 0.132 & 0.0178 & 0.0079 & 0.0032 & 0.0048 \\
\hline 6 & 0.272 & 0.270 & 0.246 & 0.261 & 6.09 & 0.101 & 0.0174 & 0.0077 & 0.0040 & 0.0035 \\
\hline 7 & 0.280 & 0.244 & 0.234 & 0.231 & 6.38 & 0.118 & 0.0201 & 0.0065 & 0.0037 & 0.0087 \\
\hline Mean & 0.268 & 0.265 & 0.213 & 0.244 & 6.51 & 0.116 & 0.0170 & 0.0075 & 0.0038 & 0.0049 \\
\hline SD & 0.051 & 0.068 & 0.052 & 0.051 & 1.56 & 0.034 & 0.0020 & 0.0014 & 0.0006 & 0.019 \\
\hline Coefficient of variation & $19.2 \%$ & $25.8 \%$ & $24.4 \%$ & $20.8 \%$ & $23.9 \%$ & $29.0 \%$ & $11.6 \%$ & $19.4 \%$ & $17.0 \%$ & $38.3 \%$ \\
\hline
\end{tabular}

Units for $K_{1}$ are $\mathrm{mL}$ blood $\mathrm{mL}^{-1}$ tissue $\mathrm{min}^{-1}$. Units for $k_{3}$ and $k_{4}$ are $\mathrm{min}^{-1}$. 
TABLE 3

VAChT Binding Estimates

\begin{tabular}{|c|c|c|c|c|c|c|c|}
\hline \multirow[b]{2}{*}{ Subject } & \multicolumn{4}{|c|}{$B P_{\mathrm{ND}}\left(\right.$ from $\left.k_{3} / k_{4}\right)$} & \multicolumn{3}{|c|}{ DVR (from $\left.V_{\mathrm{T}} / V_{\text {ref }}\right)\left(\sim 1+B P_{\mathrm{ND}}\right)$} \\
\hline & Striatum & Thalamus & Motor cortex & Cerebellar gray matter & Striatum & Thalamus & Motor cortex \\
\hline 1 & 25.9 & 3.84 & 1.69 & 0.98 & 13.6 & 2.45 & 1.36 \\
\hline 2 & 33.0 & 3.10 & 1.26 & 0.87 & 18.2 & 2.19 & 1.21 \\
\hline 3 & 15.2 & 2.81 & 2.12 & 0.76 & 9.2 & 2.16 & 1.77 \\
\hline 4 & 32.5 & 5.72 & 1.98 & 1.01 & 16.7 & 3.34 & 1.48 \\
\hline 5 & 27.5 & 3.71 & 1.64 & 0.67 & 17.1 & 2.82 & 1.59 \\
\hline 6 & 29.2 & 5.03 & 2.23 & 1.17 & 13.9 & 2.78 & 1.49 \\
\hline 7 & 13.5 & 2.30 & 0.74 & 0.42 & 10.2 & 2.32 & 1.23 \\
\hline Mean & 25.3 & 3.79 & 1.67 & 0.84 & 14.1 & 2.58 & 1.45 \\
\hline $\mathrm{SD}$ & 7.87 & 1.22 & 0.52 & 0.25 & 3.45 & 0.43 & 0.20 \\
\hline Coefficient of variation & $31.2 \%$ & $32.2 \%$ & $31.3 \%$ & $29.4 \%$ & $24.5 \%$ & $16.5 \%$ & $13.8 \%$ \\
\hline
\end{tabular}

Values are derived from kinetic parameter values of Table 1. $B P_{\mathrm{ND}}$ values in left side of table are calculated directly from ratio $k_{3} / k_{4}$. On right side of table, DVR $\left(1+B P_{\mathrm{ND}}\right)$ values are calculated from ratio of total volume of distribution estimates for target and reference tissues $\left(V_{\mathrm{T}} / V_{\text {ref }}\right)$.

delay between the arterial time course and the tracer arrival in the brain. Using these constraints makes the assumption that the nondisplaceable distribution $\left(K_{1} / k_{2}\right)$ and the dissociation rate of ${ }^{18} \mathrm{~F}$ FEOBV $\left(k_{4}\right)$ are regionally invariant. The quality of fits was similar in all 7 subjects and across regions. Kinetic analysis (Table 2) showed $K_{1}$ values of $0.2-0.3 \mathrm{~mL} \mathrm{~mL}-1 \mathrm{~min}^{-1}$ in cortical regions, suggesting first-pass extraction in the range of $25 \%-35 \%$. $V_{\mathrm{ND}}$ estimates averaged 6.5 and ranged from 4.2 to 9.4. The dissociation rate constant $\left(k_{4}\right)$ ranged from $0.003-0.009 \mathrm{~min}^{-1}$, slow, but rapid enough that the assumption of irreversible binding $\left(k_{4}=0\right)$ could not be made. The average $k_{3}$ estimates ranged from $0.116 \mathrm{~min}^{-1}$ in the striatum to $0.0036 \mathrm{~min}^{-1}$ in the occipital cortex, with corresponding $B P_{\mathrm{ND}}\left(k_{3} / k_{4}\right)$ values of 25.3 and 0.84 , respectively (Table 3 , left). $V_{\mathrm{T}}$ measures $\left(K_{1} /\left[k_{2}\left(1+k_{3} / k_{4}\right)\right]\right)$ yielded a dynamic range of approximately 15 but without major reduction in intrasubject variability (data not shown). Kinetic $V_{\mathrm{T}}$ measures relative to a reference tissue (cerebellar gray matter) are given on the right side of Table 3. Because there are no brain regions devoid of VAChT binding sites, including our selected reference tissue cerebellar gray matter, the nondisplaceable binding potential $B P_{\mathrm{ND}}$ cannot be calculated without bias when using standard reference tissue methods. Hence, the table values have been left as DVR $\left(\sim 1+B P_{\mathrm{ND}}\right)$. Because of this, the $B P_{\mathrm{ND}}$ estimates derived directly from the ratio $k_{3} / k_{4}$ are considerably higher but also more variable than DVR estimates derived from total volumes of distribution. Thus, normalization to cerebellar gray matter, in comparison to direct estimates of $B P_{\mathrm{ND}}$, provides estimates of VAChT that have a compressed dynamic range but that are more precise.

\section{Reference Tissue Models and Late Static Scanning}

Estimates of DVR or $\left(1+B P_{\mathrm{ND}}\right)$ can be obtained without the use of arterial blood samples by standard reference tissue modeling. When cerebellar gray matter was used as the reference tissue, sRTM2 yielded DVR $\left(1+B P_{\mathrm{ND}}\right)$ estimates shown in Table 4. DVR gave similar results, yielding correlations across regions relative to sRTM2 of greater than 0.995 and greater than 0.984, respectively. Also reported are the tissue concentration ratios relative to cerebellar gray from the late summed images. VAChT binding indices estimated from sRTM2 yielded 20\%-25\% higher contrast than late static imaging across the range of VAChT values, with basal ganglia sRTM2 DVR consistently higher and cortical DVR tending to be slightly lower than late static image measures (Fig. 7; Table 4). The correlation of the group means between RTM and late summed images across regions was 0.993 . Regional coefficients of variation across subjects using sRTM2 were slightly smaller than those obtained using the simple late static approach in the lower binding regions, whereas they were slightly higher in the caudate nucleus and putamen.

\section{DISCUSSION}

To our knowledge, we report the first-in-human PET studies with the VAChT tracer ${ }^{18} \mathrm{~F}-\mathrm{FEOBV}$. These studies indicate that ${ }^{18} \mathrm{~F}-\mathrm{FEOBV}$ administration is safe, that ${ }^{18} \mathrm{~F}$-FEOBV brain distribution is consistent with the distribution of cholinergic terminals, and that the tracer can provide an index of VAChT binding site density. Although PET imaging after $240 \mathrm{MBq}$ $(6.5 \mathrm{mCi})$ of ${ }^{18} \mathrm{~F}-\mathrm{FEOBV}$ was sufficient to produce excellentquality PET images, preliminary human dosimetry calculations from 3 subjects demonstrate that up to $450 \mathrm{MBq}(\sim 12 \mathrm{mCi})$ could be administered while keeping radiation doses below U.S. regulatory limits for research volunteers. No adverse events or significant alterations from baseline biochemistry and hematology laboratory values were noted at mass dosages up to $1.3 \mu \mathrm{g}$.

Brain imaging revealed ${ }^{18} \mathrm{~F}$-FEOBV distribution patterns consistent with the known distribution of cholinergic terminals with highest binding in the striatum, followed by the thalamus, cerebellar vermis, amygdala, hippocampus, cerebral cortex, and cerebellar hemispheres. Low ${ }^{18} \mathrm{~F}-\mathrm{FEOBV}$ binding in the lateral cerebellar cortex contrasts with the relatively high cerebellar cortical AChE activity observed in prior human studies (13). Another interesting locus of relatively high ${ }^{18} \mathrm{~F}-\mathrm{FEOBV}$ binding is at the mesopontine junction, which likely represents localization of the PPN complex. There is also relatively high ${ }^{18} \mathrm{~F}-\mathrm{FEOBV}$ binding in the medulla, which probably correlates with the known termination zones of caudally projecting cholinergic PPN neurons (14). 


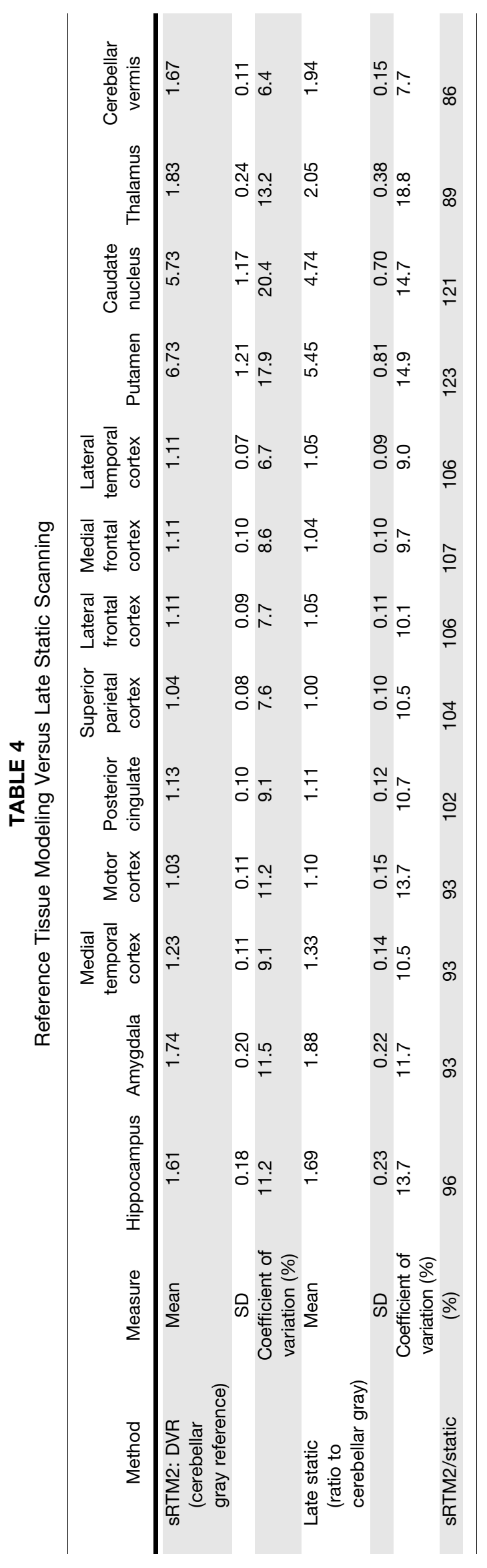

The regional distributions of brain ${ }^{18} \mathrm{~F}-\mathrm{FEOBV}$ binding strongly suggest that ${ }^{18} \mathrm{~F}-\mathrm{FEOBV}$ represents a significant advance in our ability to identify and quantify CNS cholinergic terminals. The relatively low ${ }^{18} \mathrm{~F}$-FEOBV binding in the lateral cerebellar cortex, coupled with the ability to accurately resolve ${ }^{18} \mathrm{~F}-\mathrm{FEOBV}$ binding in relatively small brain stem regions, indicates that ${ }^{18} \mathrm{~F}-\mathrm{FEOBV}$ is a significant improvement over prior VAChT methods using ${ }^{123} \mathrm{I}$-IBVM SPECT. ${ }^{18} \mathrm{~F}$-FEOBV provides more direct information about cholinergic terminal integrity than do AChE-based PET methods. AChE-based methods are indirect markers of cholinergic terminal integrity because they reflect the activity of a hydrolytic enzyme whose activity may be regulated. In addition, AChE may have both pre- and postsynaptic expressions. One disadvantage of current AChE ligands is that estimation of striatal cholinergic nerve terminal density is flow-limited because of the high striatal expression of AChE. ${ }^{18} \mathrm{~F}-\mathrm{FEOBV}$, although exhibiting high striatal uptake, is less flow-limited because FEOBV binding is not irreversible, possibly allowing a more accurate assessment of striatal cholinergic terminals.

Kinetic modeling estimates for ${ }^{18} \mathrm{~F}-\mathrm{FEOBV}$ were found to be reasonably stable but required long scan times, particularly for striatal regions. Because parameter estimation was performed while shortening the duration of image data used to $120 \mathrm{~min}$ or less (data not shown), VAChT binding in striatum was progressively underestimated. When only $90 \mathrm{~min}$ of data were used, for example, $B P_{\mathrm{ND}}$ estimates in striatum were lower by approximately $35 \%$, whereas the across-subject coefficient of variation increased by more than $40 \%$. Estimates of $B P_{\mathrm{ND}}$ in the thalamus and cerebellar vermis were found to be stable when using $120 \mathrm{~min}$ of data, whereas estimates in cortical regions were stable using $90 \mathrm{~min}$ of data. Parameter estimates in regions with the lowest VAChT density, such as the visual cortex, could be estimated accurately with as little as $60 \mathrm{~min}$ of data. The best indices of VAChT binding density were found to be either direct estimation of $B P_{\mathrm{ND}}\left(k_{3} / k_{4}\right)$ or the total volume of distribution, $V_{\mathrm{T}}$. The normalization of kinetic $V_{\mathrm{T}}$ values by $V_{\mathrm{T}}$ of cerebellar gray to yield DVR (or the indirect measure of 1 $+B P_{\mathrm{ND}}$ ) reduced variance to some degree, as seen in Table 3 , but more so in regions with lower VAChT binding densities than in the striatum.

Because full kinetic modeling with arterial sampling and plasma metabolite analysis is not practical on a routine basis, simpler reference tissue methods and late static imaging approaches were evaluated. These methods were found to yield lower variance in VAChT binding estimates but with reduced dynamic range. When only $120 \mathrm{~min}$ of data instead of the entire image sequence were used, binding estimates from sRTM were relatively unchanged, although slightly more variable in all regions except the striatum, which, like the compartmental analysis, resulted in an underestimation of binding densities. When $120 \mathrm{~min}$ of image data and a fixed value for $k_{2}{ }^{\prime}\left(k_{2}\right.$ of the reference tissue; sRTM2) were used, voxelwise parametric images of DVR $\left(1+B P_{\mathrm{ND}}\right)$ with excellent noise properties were generated. Estimates of $k_{2}{ }^{\prime}$ ranged from about 0.02 to $0.04 \mathrm{~min}^{-1}$. The fixed value of $k_{2}{ }^{\prime}$ had little effect on the magnitude of DVR estimates in all regions, again except the striatum, where the smaller $k_{2}{ }^{\prime}$ value resulted in considerably higher DVR estimates and hence a greater dynamic range but also with greater variability. When the higher value of $0.02 \mathrm{~min}^{-1}$ was used, DVR estimates were more precise but showed greater underestimates in 


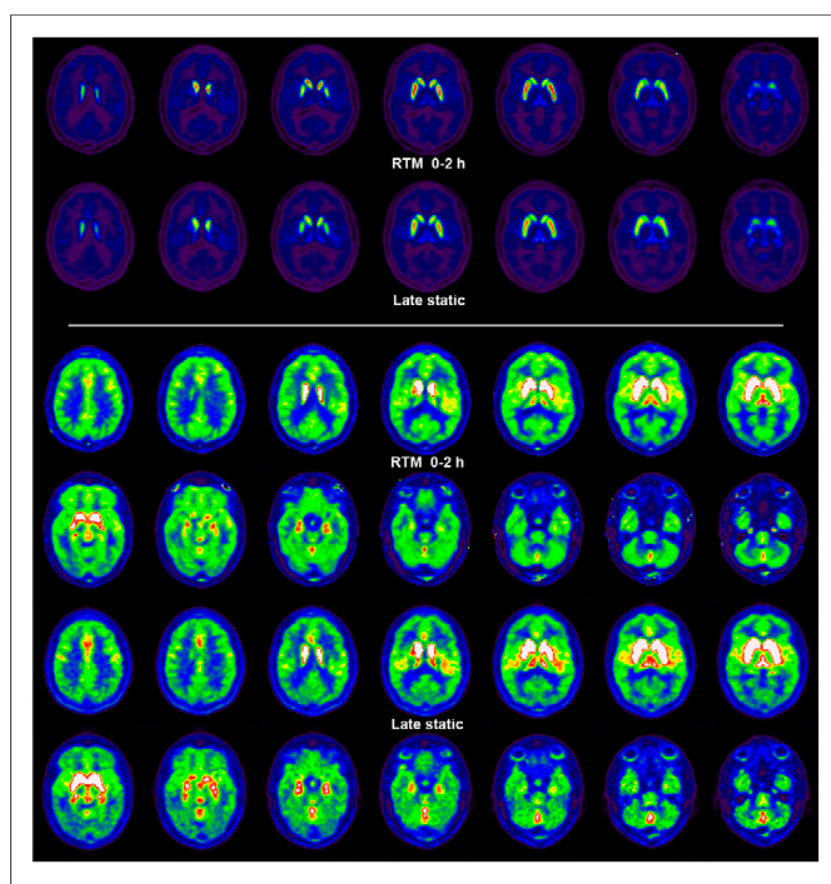

FIGURE 7. Parametric images of $1+B P_{\mathrm{ND}}$ from 120 min of imaging (rows $1,3,4$ ) and late static imaging (rows 2, 5, 6). Display maximum is 9.0 for top 2 rows and 2.25 for bottom 4 rows.

striatum, compared with the results obtained with measured arterial input function compartmental modeling. Reference tissue modeling of ${ }^{18} \mathrm{~F}-\mathrm{FEOBV}$ is able to provide a stable index of VAChT binding site density but one that trades off increased bias for better precision.

A simple late static scan of ${ }^{18} \mathrm{~F}-\mathrm{FEOBV}$ was seen to correlate highly with RTM modeling of $120 \mathrm{~min}$ of scan data. The advantages of static imaging are obvious in that only short scan durations ( $\sim 30-40 \mathrm{~min})$ are required and no kinetic analysis is necessary, much in the same manner as highly useful static ${ }^{18} \mathrm{~F}$ FDG imaging has proved incredibly valuable. The primary disadvantage is that in the slowly equilibrating striata, there is some sensitivity to changes in the tracer uptake rate, $K_{1}$, which in turn is related to change in blood flow. Computer simulations, using typical rate constant values for FEOBV from Table 2 , show that decreases in cerebral blood flow of $20 \%$ (changes in both $K_{1}$ and $k_{2}$ ) in the striatum, with $k_{3}$ unchanged, result in only a $3.5 \%$ reduction in the striatal values of the static image 3.5-4.0 $\mathrm{h}$ after injection (assuming no change in either flow or $k_{3}$ of the reference tissue). This flow-related bias is smaller in regions with lower VAChT density, where isolated $20 \%$ flow reductions in the thalamus and occipital cortex (without change in $k_{3}$ ) result in late static image changes of only $-0.6 \%$ and $+0.1 \%$, respectively.

Our results suggest some intriguing new findings about the organization of cholinergic pathways in the human CNS. Our data suggest differential cholinergic innervation of different neocortical regions, with anterior cingulate and primary sensorimotor cortices receiving disproportionate cholinergic innervation. These results suggest a particularly important role for cholinergic neurotransmission in these subregions. There are similar intriguing findings in the cerebellar cortex. We found relatively high ${ }^{18}$ F-FEOBV binding in the superior vermis and in the flocculonodular lobes.
There is only modest prior literature on cholinergic innervation of the cerebellum, which does not clearly describe a strong vermal innervation, though early studies based on acetylcholinesterase histochemistry in rat brain suggest preferential cholinergic vermal innervation $(15,16)$. It is possible that superior vermal ${ }^{18}$ F-FEOBV binding represents terminals of a PPN projection relatively specific to primates. Prior reports indicate a substantial projection from the deep cerebellar nuclei to the PPN that may be unique to primates (17). Recent functional imaging studies of human gait indicate a strong functional connection between the PPN and midline cerebellar structures (18). The superior vermal cerebellar cortex plays a particularly important role in maintenance of normal posture and gait, primarily related to somatosensory feedback. Normal PPN function is crucial also for normal posture and gait. It is plausible that increased anatomic and functional connections between the PPN and the cerebellar vermis are linked to bipedalism in humans. Similarly, our results suggest an important cholinergic innervation of the flocculonodular lobes. These are likely to be cholinergic projections from the vestibular nuclei, which are highly interconnected with this phylogenetically ancient portion of the cerebellum (15). The vestibular nuclei and their cerebellar connections are also important in the maintenance of posture and gait.

\section{CONCLUSION}

The data show that ${ }^{18} \mathrm{~F}$-FEOBV has acceptable dosimetry and safety profiles in human subjects and that it binds to VAChT with good sensitivity and specificity. Although the time and effort required to obtain PET scans that allow full compartmental kinetic analysis are prohibitive, shorter simpler scans using reference tissue modeling (90-120 $\mathrm{min}$ in duration), or even simple late static scanning (30-40 min in duration around 3-4 h after injection), correlate well with the results from longer and more complex scanning methods. ${ }^{18} \mathrm{~F}-\mathrm{FEOBV}$ has distinct advantages over currently available cholinergic imaging agents, predominantly in its ability to provide a robust index presynaptic cholinergic nerve terminals. This is of particular interest given the disruptions in the cholinergic system observed in several neurologic diseases.

\section{DISCLOSURE}

The costs of publication of this article were defrayed in part by the payment of page charges. Therefore, and solely to indicate fact, this article is hereby marked "advertisement" in accordance with 18 USC section 1734 . No potential conflict of interest relevant to this article was reported.

\section{REFERENCES}

1. Kuhl DE, Koeppe RA, Fessler JA, et al. In vivo mapping of cholinergic neurons in the human brain using SPECT and IBVM. J Nucl Med. 1994;35: 405-410.

2. Kuhl DE, Minoshima S, Fessler JA, et al. In vivo mapping of cholinergic terminals in normal aging, Alzheimer's disease and Parkinson's disease. Ann Neurol. 1996;40:399-410.

3. Albin RL, Cross D, Cornblath WT, et al. Diminished striatal $\left[{ }^{123} \mathrm{I}\right]$ iodobenzovesamicol binding in idiopathic cervical dystonia. Ann Neurol. 2003;53:528532.

4. Lewis PR, Shute CC, Silver A. Confirmation from choline acetylase analyses of a massive cholinergic innervation to the rat hippocampus. J Physiol. 1967;191: $215-224$. 
5. Henderson Z, Greenfield SA. Ultrastructural localization of acetyl-cholinesterase in substantia nigra: a comparison between rat and guinea pig. J Comp Neurol. 1984;230:278-286.

6. Mulholland GK, Wieland DM, Kilbourn MR, et al. $\left[{ }^{18} \mathrm{~F}\right]$ fluoroethoxybenzovesamicol, a PET radiotracer for the vesicular acetylcholine transporter and cholinergic synapses. Synapse. 1998;30:263-274.

7. Kilbourn MR, Hockley B, Lee L, et al. PET imaging of (2R,3R)-5- $\left[{ }^{18} \mathrm{~F}\right]$ fluoroethoxybenzovesamicol ((-)-FEOBV) in rat and monkey brain: a radioligand for the vesicular acetylcholine transporter. Nucl Med Biol. 2009;36:489493.

8. Shao X, Hockley BG, Tluczek LJM, Hoareau R, Scott PJH. Highlighting the versatility of the Tracerlab synthesis modules. Part 1: fully automated production of $\left[{ }^{18}\right.$ F]labelled radiopharmaceuticals using a Tracerlab FXFN. J Labelled Comp Radiopharm. 2011;54:292-307.

9. Stabin MG, Sparks RB, Crowe E. OLINDA/EXM: the second-generation personal computer software for internal dose assessment in nuclear medicine. $\mathrm{J} \mathrm{Nucl}$ Med. 2005;46:1023-1027.

10. Logan J, Fowler JS, Volkow ND, Wang GJ, Ding YS, Alexoff DL. Distribution volume ratios without blood sampling from graphical analysis of PET data. J Cereb Blood Flow Metab. 1996;16:834-840.
11. Lammertsma AA, Hume SP. Simplified reference tissue model for PET receptor studies. Neuroimage. 1996;4:153-158.

12. Ichise M, Liow JS, Lu JQ, et al. Linearized reference tissue parametric imaging methods: application to $\left[{ }^{11} \mathrm{C}\right] \mathrm{DASB}$ positron emission tomography studies of the serotonin transporter in human brain. J Cereb Blood Flow Metab. 2003;23:10961112 .

13. Kuhl DE, Koeppe RA, Minoshima S, et al. In vivo mapping of cerebral acetylcholinesterase activity in aging and Alzheimer's disease. Neurology. 1999;52:691-699.

14. Martinez-Gonzalez C, Bolam JP, Mena-Segovia J. Topographical organization of the pedunculopontine nucleus. Front Neuroanat. 2011;5:22.

15. Jaarsma D, Ruigrok TJ, Caffé R, et al. Cholinergic innervation and receptors in the cerebellum. Prog Brain Res. 1997;114:67-96.

16. Shute CC, Lewis PR. Cholinesterase-containing pathways of the hindbrain; afferent cerebellar and centrifugal cochlear fibers. Nature. 1965;205:242-246.

17. Hazrati LN, Parent A. Projection from the deep cerebellar nuclei to the pedunculopontine nucleus in the squirrel monkey. Brain Res. 1992;585: 267-271.

18. Jahn K, Zwergal A. Imaging supraspinal locomotor control in balance disorders. Restor Neurol Neurosci. 2010;28:105-114. 